\title{
Prospects of measuring Neutral Gauge Boson Couplings with the ATLAS detector
}

\author{
Eyal Brodet \\ Tel-Aviv University
}

\begin{abstract}
Prospects of measuring Neutral Triple Gauge Boson Couplings (NTGC) in $q \bar{q} \rightarrow Z Z \rightarrow 4$ leptons (muons and electrons) events in ATLAS are presented. This is done through reconstructing the Transverse momentum $\left(P_{t}\right)$ distribution of the $\mathrm{Z}$ Boson. As a cross check a variable including all the relevant kinematics information called the optimal observable was used. The limits on possible NTGC are extracted by binning the $P_{t}$ distribution and using Poisson statistics to make a likelihood fit. The results presented correspond to an integrated luminosity of $100 \mathrm{fb}^{-1}$ and used events passing the full ATLAS simulation program.
\end{abstract}




\section{Introduction}

The Standard Model (SM) has precise prediction for the production of ZZ events through $q \bar{q}$ interaction as shown in figure 1. It may be the case that there are non-SM direct triple gauge couplings that also produce ZZ events [1]. The ZZ process was studies in LEP2 and limits for the couplings were obtained [2]:

$$
\begin{array}{ll}
-0.3<f_{4}^{Z}<0.3 & -0.17<f_{4}^{\gamma}<0.19 \\
-0.34<f_{5}^{Z}<0.38 & -0.32<f_{5}^{\gamma}<0.36
\end{array}
$$

The LEP2 study was limited by poor statistics and the energy of the machine. In the LHC these limits are expected to significantly improve. The relatively large number of ZZ events expected in the LHC and thier high energies will give sensitivity not available before to possibly observe such couplings, and if no such couplings are found, to put better limits on their existence. The events high energy in the LHC is of a particular importance as the non-SM couplings are expected to be very sensitive to the events energy. Previous work on this subject at ATLAS was done in an ATLAS note [5]. However this was done only with fast simulation of the detector. This work is done with full simulation. At Fermilab there was no direct study of this topic due to poor statistics but there were closely related studies for example on $W W \gamma$ and on $\mathrm{ZZ} \gamma$ $[7],[8]$. In these studies no deviation from the SM was found.

The paper is organized as follows. Section 2 presents the theoretical framework. In section 3 the event generation and software are discussed. Section 4 presents the event selection and proposed measurement technique. The systematic uncertainties are discussed in section 5 and the results are presented in section 6 .

\section{Theoretical Framework}

\section{1 $Z^{*} Z Z$ and $\gamma^{*} Z Z$ Anomalous couplings}

At the SM $Z Z$ production proceeds through the Feynman diagrams shown in figure 1. For the non-SM case, the production may proceeds through the diagram shown in figure 2. As can be seen from figure 2 in the case of Neutral Triple Gauge Coupling (NTGC) there is a vertex between the 3 gauge bosons. The most general vertex function is given by [1]:

$$
\Gamma_{Z Z V}^{\alpha \beta \mu}=\frac{\hat{s}-M_{V}^{2}}{M_{Z}^{2}}\left(1 f_{4}^{V}\left(P^{\alpha} g^{\mu \beta}+P^{\beta} g^{\mu \alpha}\right)+1 f_{5}^{V} \varepsilon^{\mu \alpha \beta \rho}(q 1-q 2)_{\rho}\right)
$$

where $f_{4}^{V}$ and $f_{5}^{V}$ are the anomalous couplings for the vertex, $V$ stands for a $Z^{*}$ or a $\gamma^{*}, q 1$ and $q 2$ are the 4-momentum of the outgoing $Z$ 's, $P$ is the 4 -momentum of the intermediate $Z^{*}$ or $\gamma^{*}, \hat{s}$ is the invariant mass of the 4 leptons. The couplings $f_{4}^{V}$ is $\mathrm{CP}$ violating while $f_{5}^{V}$ is $\mathrm{CP}$ conserving but violates parity. This vertex factor is not gauge invariance. 

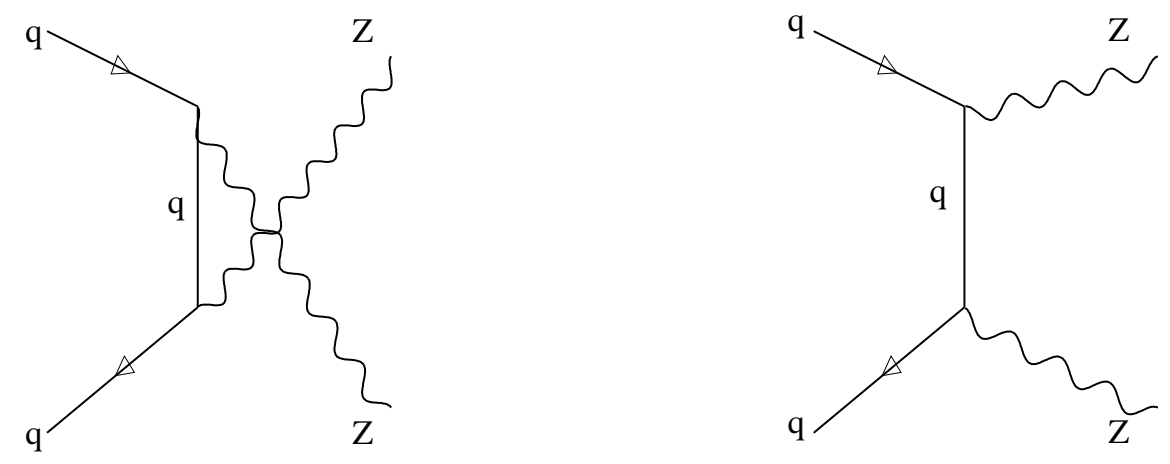

Figure 1: ZZ production in the SM.

\subsection{Form factor}

As can be seen from equation 1 the presence of $\hat{s}$ in the vertex function may, at high values, reach unitarity-violating size. In order to work around this problem a correction factor is introduced in the expression for the couplings. This addition is called a form factor and the corrected couplings is given by:

$$
f_{i}^{V}=\frac{f_{i 0}^{V}}{\left(1+\frac{\hat{s}}{\Lambda_{F F}^{2}}\right)^{n}}
$$

where $\Lambda_{F F}$ is the energy scale for the interaction, $f_{i 0}^{V}$ is the bare coupling and $\hat{s}$ is the events energy obtained from the invariant mass of the 4 leptons.

\section{Event Generation}

SM samples of simulated data events of $q \bar{q}$ producing two $Z$ 's in the final state were generated using the Pythia MC generator.

In order to evaluate the significance of possible background sources MC samples for $t \bar{t}$ and $Z b \bar{b}$ events were produced using Pythia and the Acer MC respectively. The sample sizes were 50,000 events for the signal and 100,000 events for the backgrounds. The cross section for the signal was: $158.8 \mathrm{fb}$ with a filter efficiency of 0.21 , for the $t \bar{t}$ : $708000 \mathrm{fb}$ with a filter efficiency of 0.0083 and for the $Z b \bar{b}: 49000 \mathrm{fb}$ with a filter efficiency of 0.0135 .

The generated MC samples were processed by the full ATLAS simulation program and then reconstructed as expected for real data. 


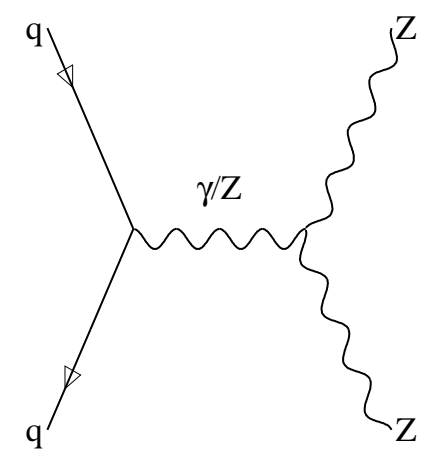

Figure 2: ZZ production through non-SM couplings.

The anomalous couplings processes are not included in Pythia MC. In order to include possible anomalous couplings contribution to the cross section the Baur [2] MC was used to give the appropriate weight to the SM Pythia events such that they will describe the presence of such couplings.

\section{Measurement of the NTGC}

\subsection{Event selection}

The process of $Z Z \rightarrow l^{+} l^{-} l^{+} l^{-}, l=e, \mu$, provides a very clean signal. The possible background sources are $t \bar{t}$ and $Z b \bar{b}$ events, both of which can contain four leptons in the final state. However, the requirement of 4 isolated leptons and that the pair of leptons invariant mass be close to the $Z$ mass reduces both of these backgrounds considerably. The mass cut also removes the $Z \rightarrow \tau \bar{\tau}$ background, as in this case the electron or muon coming from the $\tau$ will be much less energetic and will not add up to give the full $Z$ mass. The signal selection proceeds by requiring the event to have four identified isolated leptons. The identification of the leptons(muons and electrons) is done by the standard AOD identifier. The leptons are than paired into $Z$ pairs by requiring that their electric charge would be opposite and selecting the lepton combination that is closest to the $Z$ mass. In this study the cone jet algorithm is used with $\Delta R=0.4$. The full list of cuts applied are presented in table 1 . The $P_{T}$ and rapidity cuts are motivated by the ATLAS detector acceptance and trigger requirements [3]. The number of events passing each of the selection cuts for $100 \mathrm{fb}^{-1}$ is shown in table 2 . 


\begin{tabular}{|r|c|}
\hline Transverse lepton momentum & $P_{T}>7$ \\
\hline Lepton rapidity & $|\eta|<2.5$ \\
\hline$Z$ mass constraint & $\left|M_{l l}-91.2\right|<12 \mathrm{GeV}$ \\
\hline At least 2 leptons with & $P_{T}>20 \mathrm{GeV}$ \\
\hline Jet veto & $P_{T}^{\text {Jet }}<30$ \\
\hline
\end{tabular}

Table 1: The cuts applied for $q \bar{q} \rightarrow Z Z$ selection.

\begin{tabular}{|r|c|c|c|}
\hline & $Z Z$ & $Z b \bar{b}$ & $t \bar{t}$ \\
\hline 4 isolated leptons & $1053 \pm 32.4$ & $9545 \pm 138.1$ & $43870 \pm 468.3$ \\
\hline$P_{T}$ and rapidity & $758 \pm 27.5$ & $1110 \pm 47.1$ & $4510 \pm 150.1$ \\
\hline$Z$ mass constraint & $486 \pm 22.0$ & $195 \pm 19.7$ & $70 \pm 18.7$ \\
\hline Jet veto & $448 \pm 21.1$ & $4 \pm 2.8$ & $5 \pm 5$ \\
\hline
\end{tabular}

Table 2: Expected number of events for signal and background after cuts for an integrated luminosity of $100 \mathrm{fb}^{-1}$.

\subsection{Measurement technique}

The presence of NTGC are manifested as an excess of events. A good variable to observe this excess is in the $P_{t}$ distribution of the $\mathrm{Z}$ boson. This variable is sensitive to both the energy of the event and the production angle of the $Z$. The NTGC are expected to have the largest effect at high $P_{t}$ values. This can be seen in figure 3 where the cross section ratio of events with NTGC and of SM are plotted against the value of the coupling and also in figure 4 where the $\mathrm{Z} P_{t}$ is shown for SM and NTGC events.

In order to get limits on NTGC one can use the Poisson probability to construct a likelihood for these events. The Poisson probability is given by:

$$
P_{i}=\frac{e^{-\mu_{i}} \cdot \mu_{i}^{n_{i}}}{n_{i} !}
$$

where $n_{i}$ is the number of observed events in bin $\mathrm{i}$ and $\mu_{i}$ is the expected number of events for any given values of NTGC $f_{j}^{\gamma}, f_{j}^{Z}(\mathrm{j}=4,5)$ is $[4]$ :

$$
\begin{aligned}
\mu_{i}\left(f_{j}^{\gamma, Z}\right) & =\left(1-\left(\frac{f_{j}^{\gamma, Z}}{f_{j 1}^{\gamma, Z}}\right)^{2}\right) \cdot \mu_{i}(0)-\left(\frac{f_{j}^{\gamma, Z}}{2 f_{j 1}^{\gamma, Z}}-\frac{1}{2}\left(\frac{f_{j}^{\gamma, Z}}{f_{j 1}^{\gamma, Z}}\right)^{2}\right) \cdot \mu_{i}\left(-f_{j 1}^{\gamma}, Z\right) \\
& +\left(\left(\frac{f_{j}^{\gamma, Z}}{2 f_{j 1}^{\gamma, Z}}\right)-\frac{1}{2}\left(\frac{f_{j}^{\gamma, Z}}{f_{j 1}^{\gamma, Z}}\right)^{2}\right) \cdot \mu_{i}\left(f_{j 1}^{\gamma, Z}\right)
\end{aligned}
$$

Here $f_{j 1}^{\gamma}$ and $f_{j 1}^{Z}$ are templates values for $f_{j}^{\gamma}$ and $f_{j}^{Z}$ which can be chosen arbitrarily. In the 
analysis, $f_{j 1}^{\gamma}=0.1$ and $f_{j 1}^{Z}=0.1$ were set. The Likelihood is given by:

$$
L=\prod_{i=1}^{N_{b}} P_{i}
$$

where $N_{b}$ is the number of $P_{t}$ bins. The negative log Likelihood is given by:

$$
-\ln L=-\sum_{\mathrm{i}=1}^{\mathrm{N}_{\mathrm{b}}}\left[\mathrm{n}_{\mathrm{i}} \cdot \ln \left(\mu_{\mathrm{i}}\right)-\mu_{\mathrm{i}}\right]
$$

The sample is divided into 7 bins in $\mathrm{Z} P_{t}$. The fit is done only for the highest $6 P_{t}$ bins. The first $P_{t}$ bin is used for normalization of the $\mathrm{MC}$ to the data distribution using the fact that the number of events in this lowest $P_{t}$ bin is expected to be insensitive to NTGC. In this way all systematics related to the overall normalization are minimized.

In order to check how good is the sensitivity obtained from the $Z P_{t}$ variable it was compared to a variable called the Optimal Observable (OO). This variable is defined as [5]:

$$
O O_{f_{4}^{Z}}=\lim _{f_{4}^{Z} \rightarrow 0} \frac{\frac{d \sigma\left(S M+f_{4}^{Z}\right)}{d \Omega}-\frac{d \sigma(S M)}{d \Omega}}{f_{4}^{Z} \cdot \frac{d \sigma(S M)}{d \Omega}}
$$

where $\frac{d \sigma}{d \Omega}$ is the differential cross section. The OO is the relative change in the cross section for a given step in the relevant coupling. i.e. a step from the couplings SM value. This variable does not depend on the value of the coupling and only reflects the change in the cross section. This variable should have the best sensitivity to NTGC as it includes all the relevant kinematic information in the event. A distribution of the $O O_{f_{4}^{Z}}$ is shown in figure 5. One can observe the excess of events at high $O O_{4}^{Z}$ values.

\section{Systematics}

A central role of the MC in this analysis is to predict the number of expected events with a particular NTGC value. Therefore it was necessary to consider systematic effects on this number. The uncertainties considered on this numbere were MC statistics, the choice of the structure function and the uncertainty on the normalization factor. The error from MC statistics was computed by making the fit to a large MC sample where the number of observed events and the expected number is identical. In this case the likelihood is sensitive to the error due to the size of the MC sample and therefore a step associated to $68 \%$ would be an estimate of the systematic error.

The uncertainties due to the proton structure function were estimated using Baur MC from which it was possible to calculate a weight to the event with different choices of structure functions. The difference between the limits obtained due to the different structure function and the used structure function were taken as a systematic uncertainty. The error due to the 


\begin{tabular}{|r|c|c|c|c|c|c|c|}
\hline couplings & EHLQ2 & CTEQ3L & CTEQ4L & CTEQ2pL & MRST & MC stat & Norm. factor \\
\hline$f_{4}^{Z}$ & 0.00019 & 0.00015 & 0.00014 & 0.00033 & 0.00017 & 0.0015 & 0.0012 \\
\hline$f_{4}^{\gamma}$ & 0.00025 & 0.00011 & 0.00018 & 0.00054 & 0.00025 & 0.0020 & 0.0015 \\
\hline$f_{5}^{Z}$ & 0.00014 & 0.00004 & 0.00023 & 0.00042 & 0.00009 & 0.0015 & 0.0016 \\
\hline$f_{5}^{\gamma}$ & 0.00022 & 0.00014 & 0.00015 & 0.00049 & 0.0003 & 0.0020 & 0.0010 \\
\hline
\end{tabular}

Table 3: Systematic uncertainties evaluated from 5 different proton structure function models, from the available MC statistics and the normalization factor.

\begin{tabular}{|r|c|c|}
\hline couplings & $P_{t}(Z)$ & Optimal Observable \\
\hline$f_{4}^{Z}$ & {$[-0.0051,0.0051]$} & {$[-0.0050,0.0051]$} \\
\hline$f_{4}^{\gamma}$ & {$[-0.0075,0.0075]$} & {$[-0.0074,0.0075]$} \\
\hline$f_{5}^{Z}$ & {$[-0.0053,0.0055]$} & {$[-0.0052,0.0055]$} \\
\hline$f_{5}^{\gamma}$ & {$[-0.0078,0.0078]$} & {$[-0.0077,0.0078]$} \\
\hline
\end{tabular}

Table 4: $95 \%$ confidence level obtained from the $\mathrm{Z} P_{t}$ and $\mathrm{OO}$ variables for an integrated luminosity of $100 \mathrm{fb}^{-1}$.

normalization of the first $P_{t}$ bin was estimated by calculating its statistical error and adding it to the original number and remaking the fit.

Table 3 summarizes the systematic uncertainties obtained.

The total systematic uncertainty was estimated by adding all the values in quadrature. The systematic errors were incorporated in the fit by adding, in the likelihood calculations, $x \cdot \sigma$ to the coupling, where $\sigma$ is the total systematic uncertainty and $x$ is a free parameter in the fit.

\section{Results}

Table 4 shows the $95 \%$ confidence level limits of the 4 anomalous couplings using the $\mathrm{Z} P_{t}$ variable and using the Optimal Observable. A value of $6 \mathrm{TeV}$ was assumed for the form factor scale $\Lambda_{F F}$. The results are for integrated luminosity of $100 \mathrm{fb}^{-1}$. Only one NTGC is allowed to vary each time while the other couplings are set to zero, namely the SM value. The limits are obtained by taking the mean of 1000 LHC "experiments". The quoted values show great improvement from LEP2 results [2].

In figures 6-7 the $95 \%$ confidence level is shown as a function of the luminosity. It can be seen that at $100 \mathrm{fb}^{-1}$ there is a good sensitivity and the confidence level decreases slower afterwards. Figures 8 and 9 shows the log-likelihood curves for the different couplings for one LHC "experiment".

The effect of the systematic uncertainties described in the previous section on the $95 \%$ confidence level obtained on each couplings is shown in table 5 where the results include the systematics uncertainties. This results can be compared to table 4 where there is no systematics. As expected the effect of the systematic errors is to broaden the log-likelihood and to increase the limit. 


\begin{tabular}{|r|c|}
\hline couplings & $P_{t}(Z)$ \\
\hline$f_{4}^{Z}$ & {$[-0.0053,0.0054]$} \\
\hline$f_{4}^{\gamma}$ & {$[-0.0079,0.0079]$} \\
\hline$f_{5}^{Z}$ & {$[-0.0055,0.0057]$} \\
\hline$f_{5}^{\gamma}$ & {$[-0.0081,0.0081]$} \\
\hline
\end{tabular}

Table 5: 95\% confidence level obtained after including systematic effects.

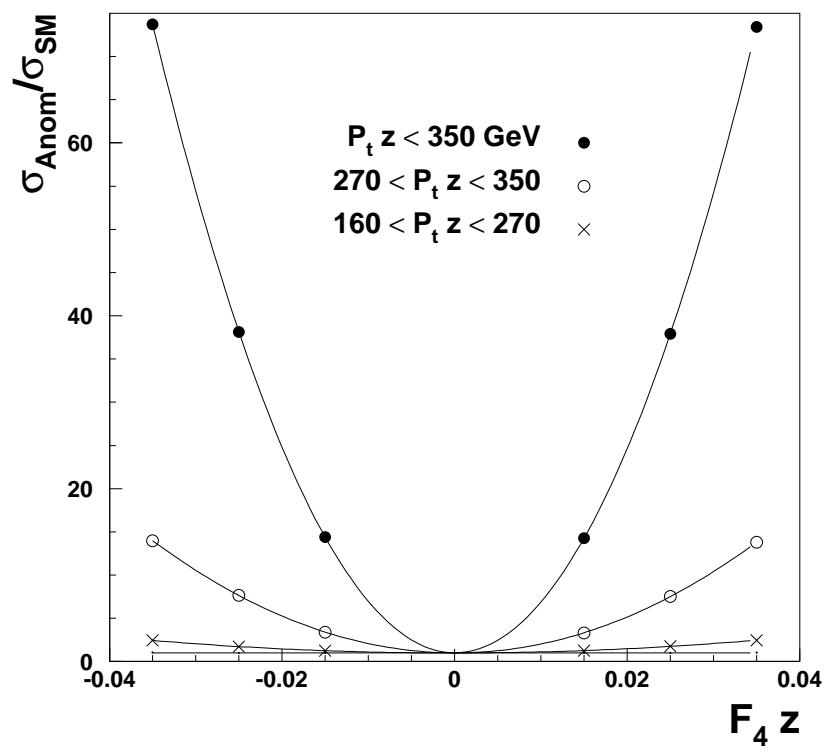

Figure 3: The Cross section ratio of with and without NTGC as a function of the $f_{Z}^{4}$ coupling for different $\mathrm{Z} P_{t}$ bins.

\section{Conclusions}

It was shown that it would be possible to obtain limits on NTGC which are a great improvement to the LEP2 result. This is due to the fact that in LHC the statistics and the energy is expected to be larger than achieved in LEP2.

As can be seen from table 2 the $\mathrm{Z} P_{t}$ is a good variable to use as there is almost no difference compared to the $\mathrm{OO}$ variable. 


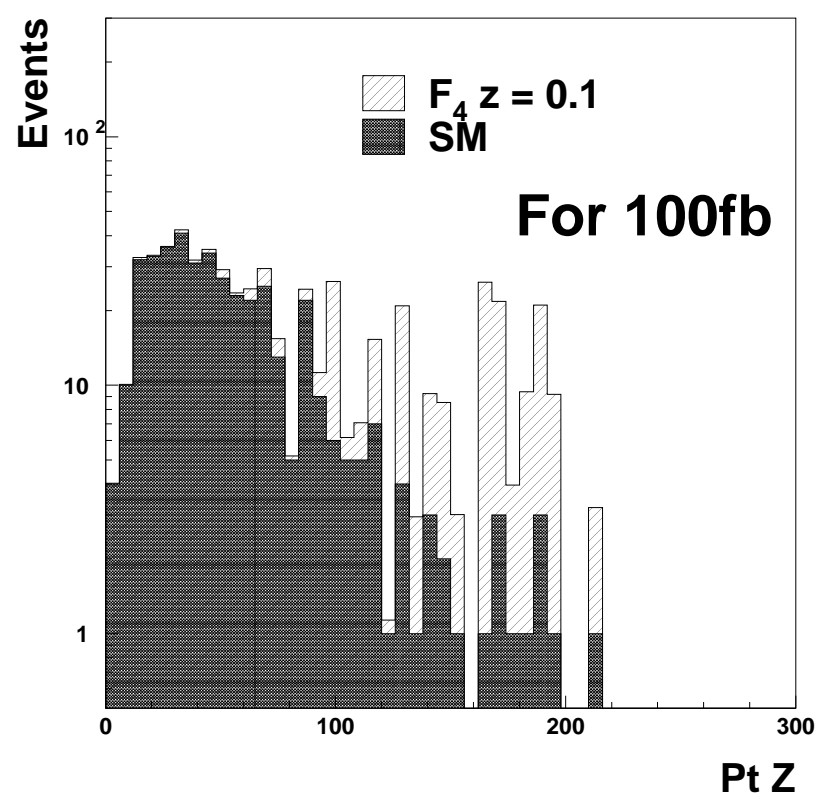

Figure 4: Distribution of the Z $P_{t}$ variable for a SM sample and for a non-SM sample corresponding to $f_{Z}^{4}=0.02$.

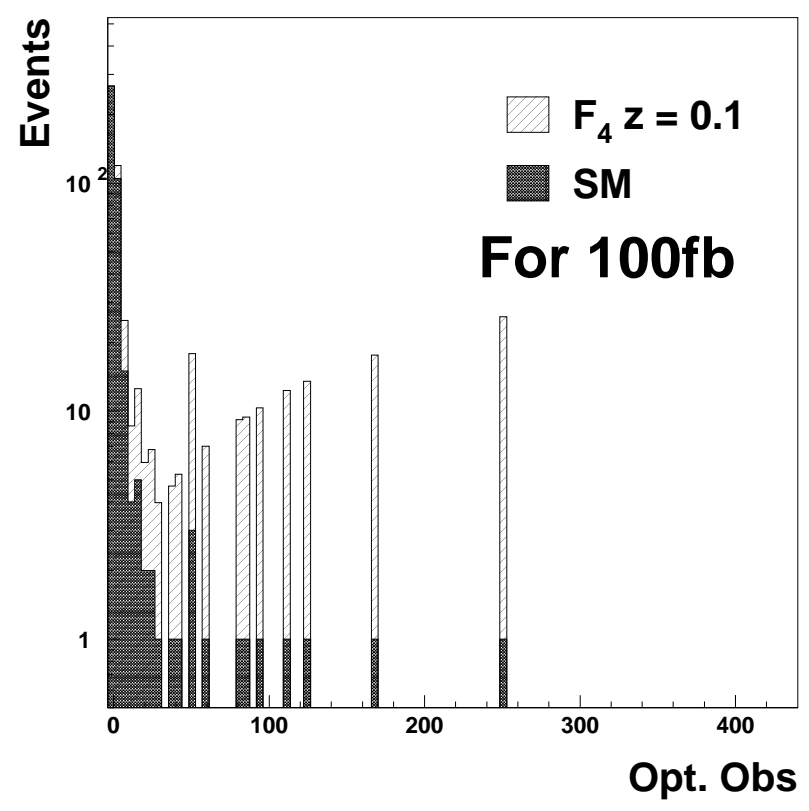

Figure 5: Distribution of the Optimal Observable $(\mathrm{OO})$ variable for a SM sample and for a non-SM sample corresponding to $f_{Z}^{4}=0.02$. 

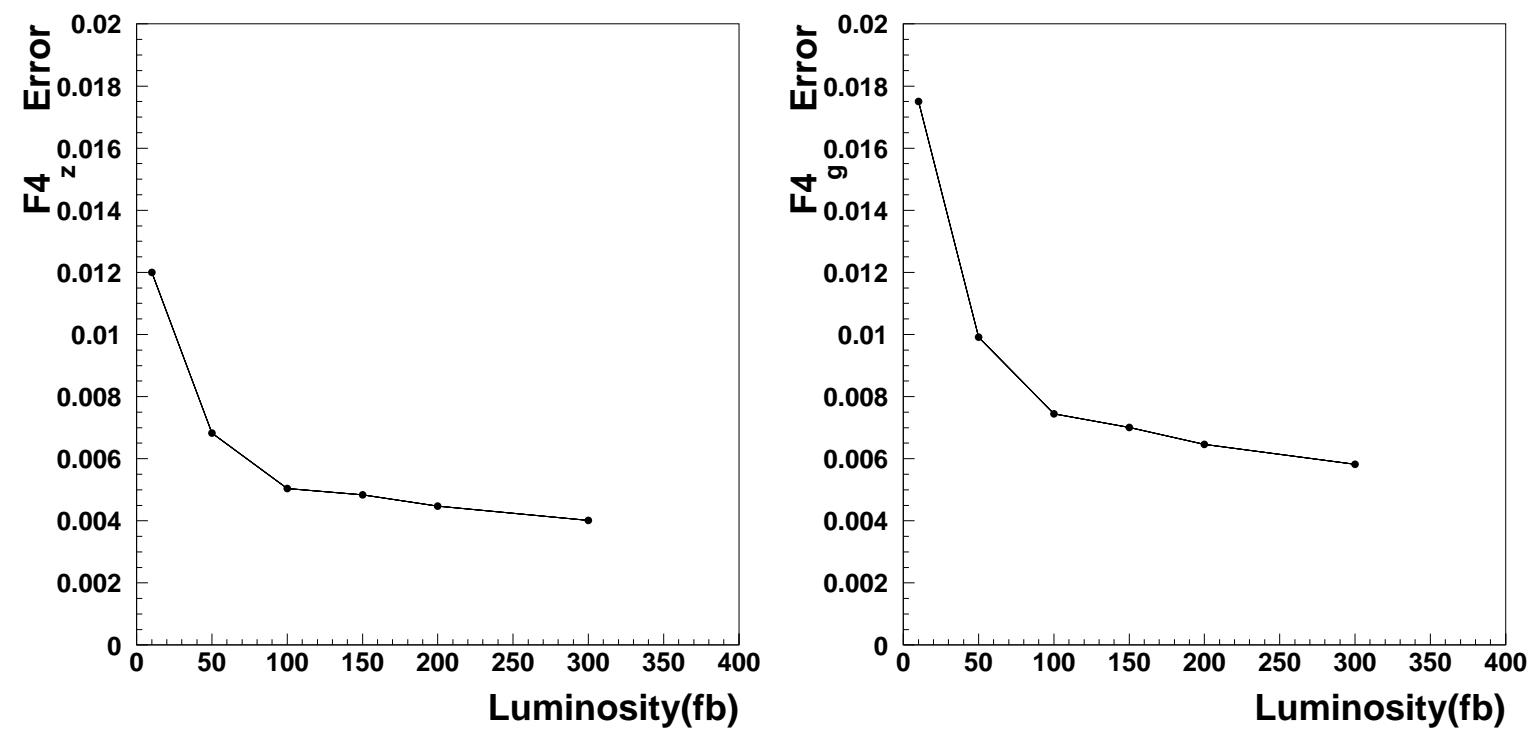

Figure 6: The $95 \%$ confidence level error of the $f_{Z}^{4}$ and $f_{\gamma}^{4}$ coupling as a function of the luminosity.
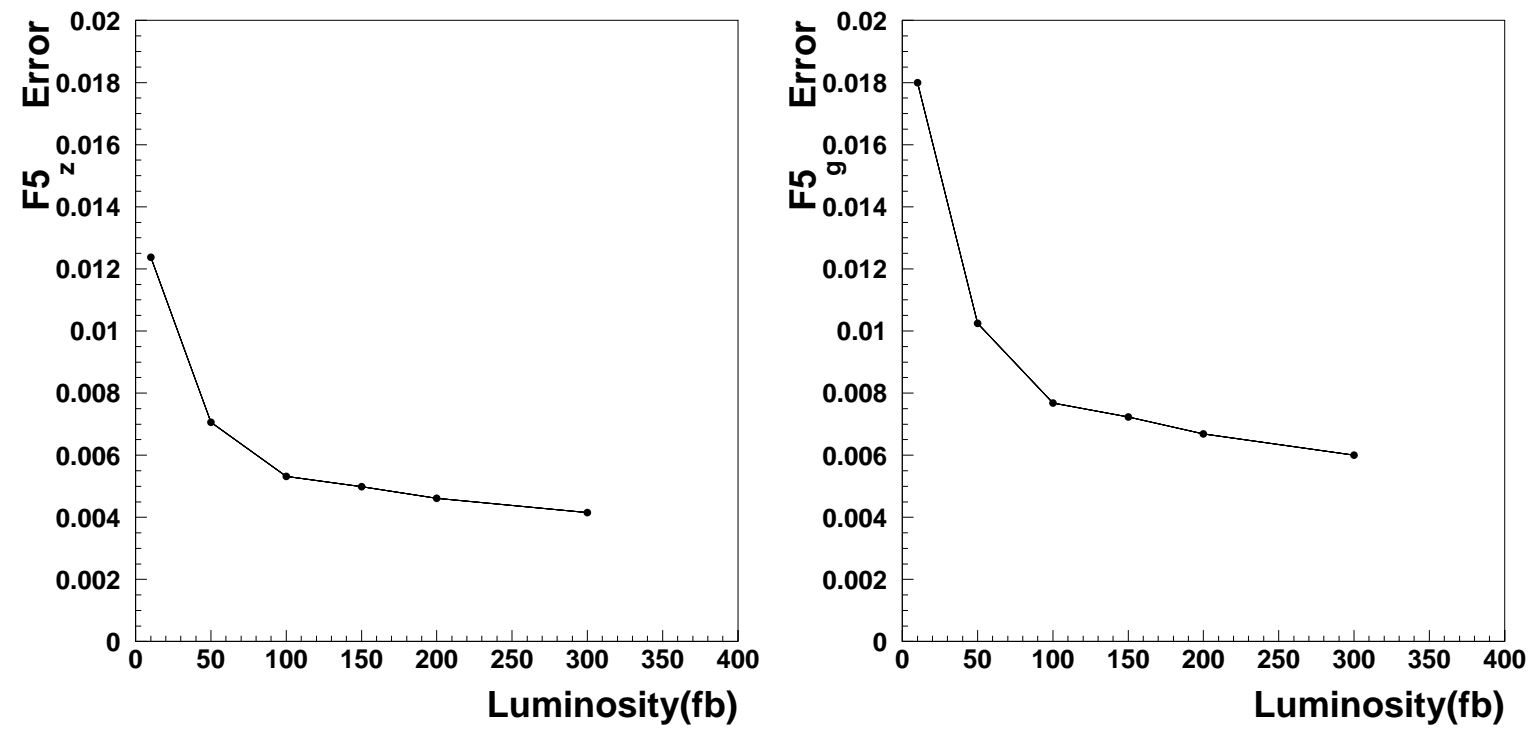

Figure 7: The $95 \%$ confidence level error of the $f_{Z}^{5}$ and $f_{\gamma}^{5}$ coupling as a function of the luminosity. 

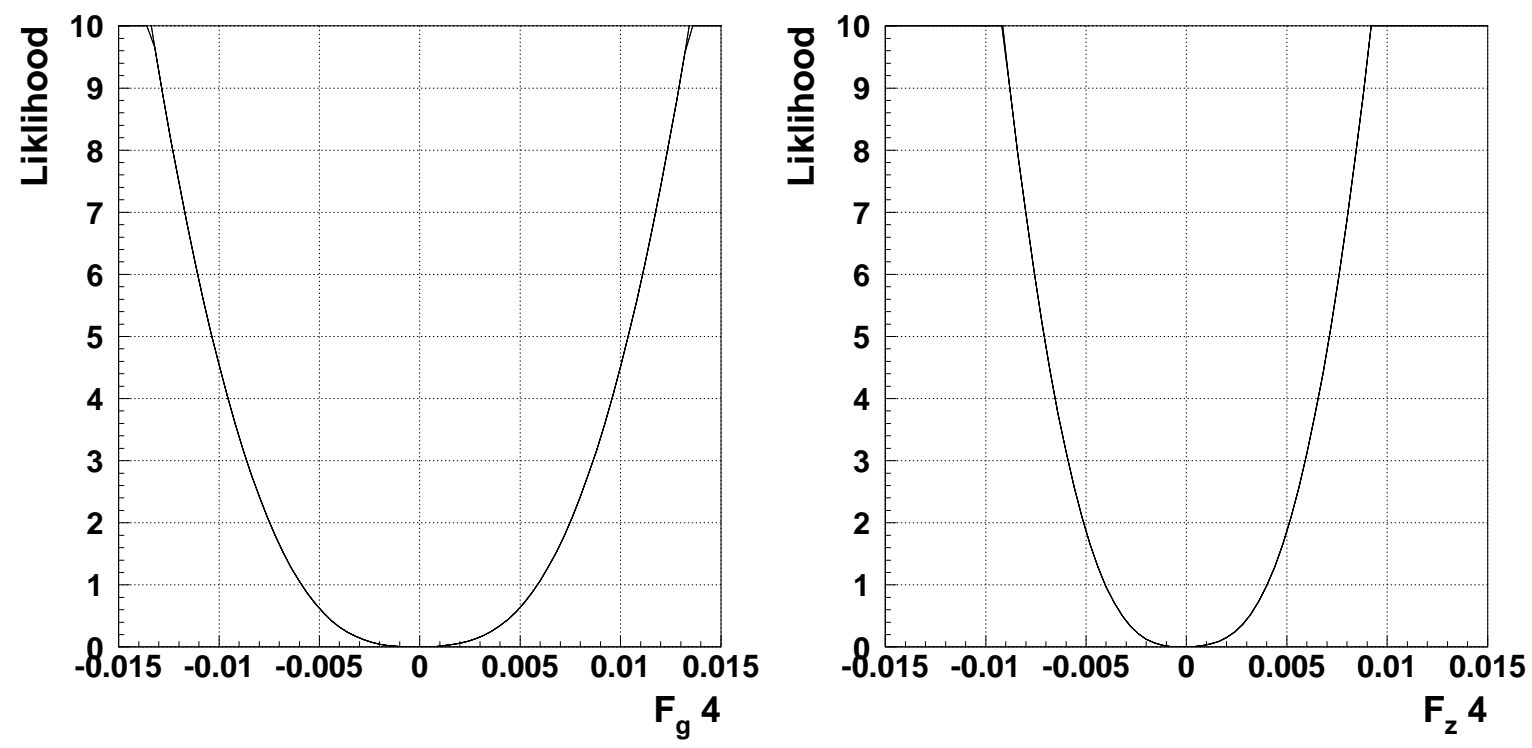

Figure 8: The log-likelihood curve for an integrated luminosity of $100 \mathrm{fb}^{-1}$ for the $f_{Z}^{4}$ and $f_{\gamma}^{4}$ coupling.
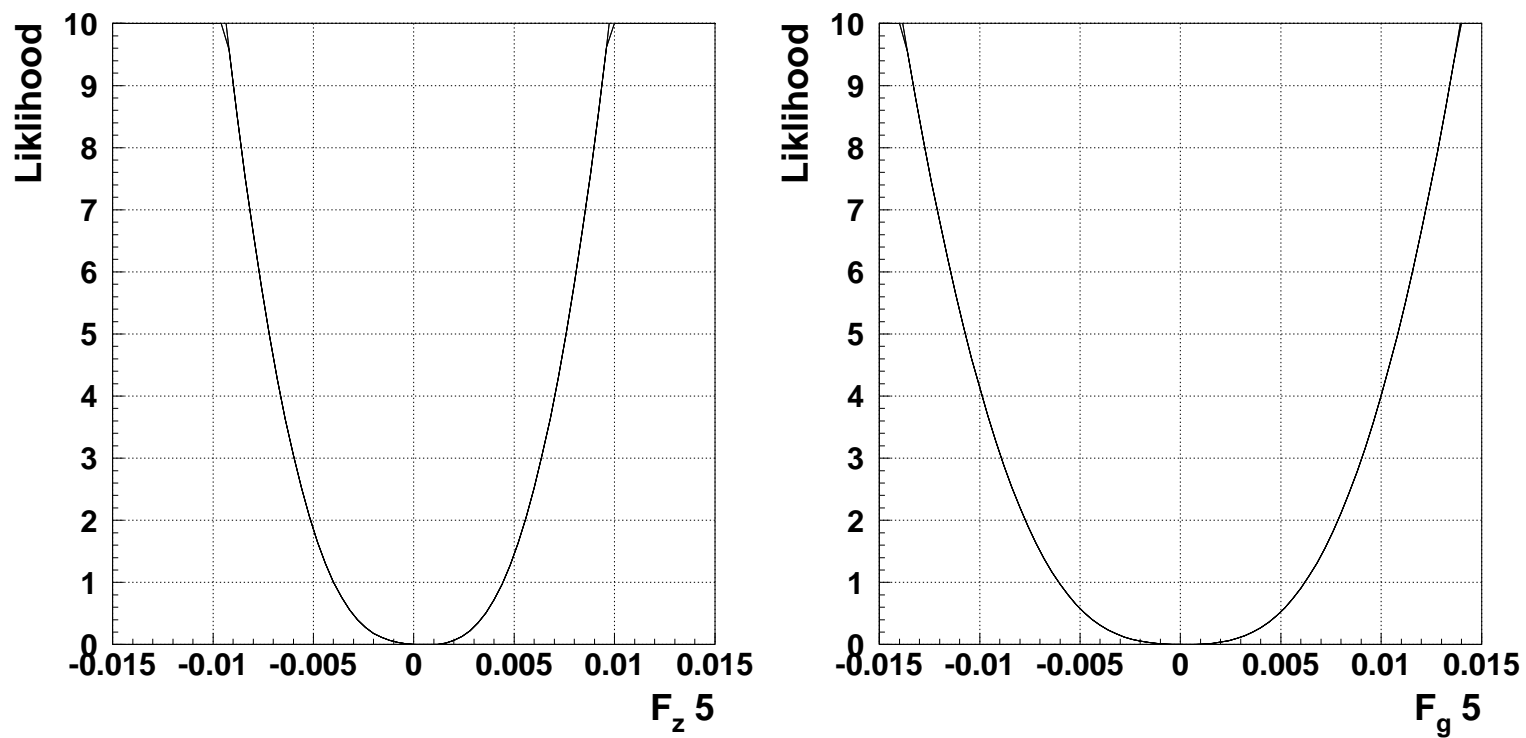

Figure 9: The log-likelihood curve for an integrated luminosity of $100 \mathrm{fb}^{-1}$ for the $f_{Z}^{5}$ and $f_{\gamma}^{5}$ coupling. 


\section{Acknowledgments}

I would like to thank Gideon Bella for many useful discussions and his invaluable guidance which were essential to the production of this note.

\section{$9 \quad$ References}

[1] K. Hagiwara, R. D. Peccei, D. Zeppenfeld, and K. Hikasa, Nucl. Phys. B282 (1987) 253.

[2] A combination of Preliminary Results on Gauge Boson Couplings Measured by the LEP Experiments. LEPEW/WG/TGC/2003-01. http://lepww.web.cern.ch/lepww/tgc/.

[3] U. Baur and D. Rainwater, Phys. Rev D62(2000)113011.

[4] ATLAS Collaboration, "Technical Proposal for a general purpose pp experiment at the Large Hadron Collider at CERN", CERN/LHCC/94-43,LHCC/P2,December 1994; "ATLAS Collaboration, "ATLAS detector and physics performance, Technical Design Report", Vol I, CERN, LHCC/99-14, ATLAS-TDR-14, May 1999.

[5] S. Hassani. ATLAS Collaboration. ATL-PHYS-2003-022.

[6] M. Dobbs, M. Lefebvre. ATLAS Collaboration. ATL-PHYS-2002-023.

[7] Dzero Collaboration. Physical Review D71,091108(R),2005.

[8] Dzero Collaboration. Fermilab-Pub-97/363-E 